\title{
Effects of Supplementation with Anti-Inflammatory Compound Extracted from Herbs in Healthy and Obese Cats
}

This article was published in the following Dove Press journal: Veterinary Medicine: Research and Reports

\author{
Motoo Kobayashi ${ }^{1,2, *}$ \\ Yuki Okada (D) ${ }^{1,2, *}$ \\ Hiromichi Ueno' \\ Takayuki Mizorogi' \\ Kenji Ohara' \\ Koh Kawasumi' \\ Kohei Suruga ${ }^{3}$ \\ Kazunari Kadokura ${ }^{3}$ \\ Yasuyuki Ohnishi $\mathbb{D}^{2}$ \\ Toshiro Arai $\mathbb{D}^{1,2}$ \\ 'Laboratory of Veterinary Biochemistry, \\ School of Veterinary Medicine, Nippon \\ Veterinary and Life Science University, \\ Tokyo I80-860I, Japan; ${ }^{2}$ One Health Co. \\ Ltd, Tokyo I57-0066, Japan; ${ }^{3}$ Food \\ Function R\&D Division, International \\ Operation Department, Kibun Foods \\ Inc., Tokyo 206-08I2, Japan \\ *These authors contributed equally to \\ this work
}

Correspondence: Toshiro Arai Laboratory of Veterinary Biochemistry, School of Veterinary Medicine, Nippon Veterinary and Life Science University, I-7-I Kyonancho, Musashino, Tokyo I80860 I, Japan

Tel $+8|4223| 4|5|$

Fax +8I 422 3I 784I

Email tarai@nvlu.ac.jp
Background: Obesity has become a serious public health problem all over the world, and prevalence of obesity has increased in cats. Obesity is characterized by continuous low-grade inflammation based on oxidative stress by excessively produced reactive oxygen species (ROS). Supplementation with anti-oxidant and anti-inflammatory compounds is very effective to relieve the obesity condition. A plant extract mixture containing Rhus verniciflua and some other herbs, Rv-PEM01-99, shows anti-oxidant and anti-inflammatory effects in animals. The aim of this study was to evaluate the effects of supplementation with RvPEM01-99 as an anti-inflammatory compound in healthy and obese cats.

Materials and Methods: Ten healthy mix breed cats and four obesity disease cats were used. The healthy cats were randomly divided into control and test groups. Antiinflammatory compound, Rv-PEM01-99, in which quercetin derivative is the main component, was supplemented to the healthy test group and the obesity disease cats at the dose of $100-120 \mathrm{mg} / \mathrm{kg} /$ day $(2.5-3.0 \mathrm{mg} / \mathrm{kg} /$ day as quercetin) for 4 weeks. Metabolites, hormones and enzymes were measured before and after the compound supplementation.

Results: The anti-inflammatory compound supplementation decreased serum amyloid A (SAA) concentrations as inflammatory markers in both healthy and obesity disease cats. In obesity disease cats, plasma total cholesterol concentrations and AST and ALT activities decreased significantly after the compound supplementation.

Conclusion: Quercetin derivative seems to have strong anti-inflammatory activities. In the healthy cats, anti-inflammatory compound supplementation decreased plasma NEFA and SAA concentrations. In the obesity disease cats, the compound supplementation may have alleviated obesity disease by relieving inflammation and improvement of lipid metabolism in livers.

Keywords: anti-inflammatory compound, cat, obesity, quercetin, SAA

\section{Introduction}

Obesity has become a serious public health problem all over the world. ${ }^{1,2}$ Obesity accompanied by visceral fat accumulation causes insulin resistance and obesity can be a risk factor for metabolic syndrome, diabetes mellitus, hypertension, dyslipidemia, and some types of cancer. ${ }^{3}$ Prevalence of obesity in dogs and cats has increased in recent years as in humans. ${ }^{4}$ Cats are more prone to become obese compared to dogs owing to their unique characteristics in glucose and lipid metabolism. ${ }^{5,6}$ Prevalence of obesity in cats is assumed to be $30 \sim 40 \%{ }^{7}$ Obesity is defined as excessive fat accumulation that presents a risk for metabolic disorders, therefore body weight reduction is required for obese animals. However, only 
weight reduction is not enough for alleviation of obesity condition in animals with accumulated visceral fat, causing systemic inflammation. ${ }^{8}$ Fat accumulation and oxidative stress impair the mitochondrial function via morphological alteration, increased membrane peroxidation, decreased ATP level, increased reactive oxygen species (ROS) production, and defective mitochondrial fatty acid $\beta$-oxidation in livers. ${ }^{9}$ Excess amount of ROS produced via accelerated $\beta$-oxidation of fatty acids is attributed to one of the pathogens for obesity and its associated diseases. ${ }^{10}$ Consequently, some antioxidant and antiinflammatory compounds appear to be effective to ameliorate obesity conditions in animals. ${ }^{11-13}$

The anti-inflammatory compound, Rv-PEM01-99, which is extracted from some kinds of herbs, shows a significant effect in improving lipid metabolism in obese dogs. ${ }^{14}$ The anti-inflammatory effect of Rv-PEM01 -99 is considered to be due to a quercetin derivative, which is a main ingredient in the compound extracted from Rhus verniciflua leaf. ${ }^{15-17}$

In this study, we investigated the changes in plasma metabolites, hormone concentrations and enzyme activities related to energy metabolism in livers of healthy and obese cats with the anti-inflammatory compound supplementation for 4 weeks. The aim of this study was to evaluate the effect of quercetin derivative as inflammatory compound on energy metabolism in healthy and obese cats.

\section{Materials and Methods}

\section{Animals}

Ten healthy control cats ( 6 female, 4 male, $2-3$ years old, mixed breed) were maintained for laboratory experiments at Narita Animal Science Laboratory Co., Ltd. (Narita, Japan) and fed on commercial diets. Four client-owned cats ( 3 female, 5, 6 and 9 years old, mixed breed and 1 castrated male, 7 years old, mixed breed) with obesity disease were entered into this study. Body weight and body condition score (BCS) was assessed. ${ }^{18}$ For overweight cats with high BCS of $>7 / 9$, showing low adiponectin $(<3.0 \mu \mathrm{g} / \mathrm{mL})$, hyperlipidemia (triglyceride $>165 \mathrm{mg} / 100 \mathrm{~mL}$ or total cholesterol $>180 \mathrm{mg} / 100 \mathrm{~mL}$ ) and high serum amyloid A concentrations $(>200 \mathrm{ng} / \mathrm{mL})$, categorized them as having obesity disease. ${ }^{8,19}$ Informed consent was obtained from each client on the written form. The informed consent included information about possible risk, benefits, and limits of examination. Ethical approval for this study was obtained from the research animal committee of Nippon Veterinary and Life Science University (No. 30K-05).

\section{Supplementation with Plant Mixture}

Rv-PEM01-99 prepared by Kibun Foods, Inc. (Tokyo, Japan) was used as the compound in this study. The amounts of each herb used for extraction was as follows: Rhus verniciflua (90 g), Ulmus hollandica (60 g), Polygonatum sibiricum (59 g), Lycium chinense (10 g), Ganoderma japonicum (10 g), Parnax ginseng (10 g), amounting to a total of $230 \mathrm{~g}$. Each of the above six herbs was ground and added to one powder (total volume $230 \mathrm{~g}$ ), which was then extracted with 10 volume of $70 \%$ ethanol in water at room temperature. The extracted solution was filtered, and the solvent was evaporated, filtered and lyophilized, yielding $60 \mathrm{~g}$ of lyophilized Rv-PEM01-99 from 230 $\mathrm{g}$ of herb powder. ${ }^{16}$ Urushiol, an allergenic substance found in Rhus verniciflua, was completely removed through the above process. Two hundred milligrams of Rv-PEM0-99 is equivalent to $5 \mathrm{mg}$ of quercetin derivative. Powdered RvPEM01-99 (300 mg) was packed in capsule form (Capsulae Operculatae, J.P No.2, Kobayashi Capsule Corporation, Hyogo, Japan), and supplemented to cats at the dose of $100120 \mathrm{mg} / \mathrm{kg} /$ day $(2.53 .0 \mathrm{mg} / \mathrm{kg} /$ day as quercetin) for 4 weeks. For example, one capsule for $3 \mathrm{~kg}$ BW cats and two capsules for $5 \mathrm{~kg} \mathrm{BW}$ cats were supplied.

\section{Blood Sampling}

Blood samples were collected before (0 week) and 4 weeks after Rv-PEM01-99 supplementation. Fasting blood (at least 10 hours after the last meal) was taken from the jugular vein and plasma and serum were separated by centrifugation with and without heparin as anticoagulant, respectively. Plasma and serum samples were stored at $-80^{\circ} \mathrm{C}$ until use.

\section{Metabolite, Hormone and Enzyme Analysis}

Plasma glucose, triglyceride (TG), total cholesterol (TC) and total protein (TP) concentrations and lactate dehydrogenase (LDH), aspartate aminotransferase (AST) and alanine aminotransferase (ALT) activities were measured using an autoanalyzer (JCA-BM2250, JEOL Ltd., Tokyo, Japan) with the manufacturer's reagents at FUJIFILM Monolith Co. Ltd. (Tokyo, Japan). Plasma non-esterified fatty acids (NEFA) concentrations were measured with a commercial kit (NEFA-C Test, Wako Pure Chemical Industries, Ltd., Tokyo, Japan). Plasma malondialdehyde (MDA) and adiponectin concentrations were measured 
with a commercial kit, NWLSSTM Malondialdehyde assay kit (Northwest Life Science Specialties, LLC, Vancouver, Canada) and mouse/rat adiponectin ELISA kit (Otsuka Pharmaceutical Co., Ltd., Tokyo, Japan), respectively. Serum amyloid A (SAA) concentrations were measured with a commercial kit, CAT SERUM AMYLOID A (SAA) ELISA (Life Diagnostics, Inc., West Chester, PA, USA)

\section{Statistics}

Measured values are expressed as means \pm standard error (SE). Statistical significance was determined by paired$t$ test. The significance level was set at $\mathrm{p}<0.05$.

\section{Results}

Comparison of biomarker levels in healthy cats with and without Rv-PEM01-99, anti-inflammatory compound, supplementation are shown in Table 1. In the healthy cats supplemented with the anti-inflammatory compound for 4 weeks, plasma NEFA, MDA and SAA concentrations and LDH and ALT activities decreased significantly. Body weight, BCS, plasma glucose, TG, TC, TP and adiponectin concentrations did not change in healthy cats after the compound supplementation. Changes of biomarker levels in four obesity disease cats with anti-inflammatory compound are shown in Table 2. The four cats were diagnosed as obesity disease by being overweight (BCS >7/9), and having low adiponectin $(<3 \mu \mathrm{g} / \mathrm{mL})$, hyperlipidemia (TC $>180 \mathrm{mg} /$
$100 \mathrm{~mL}$ ) and high SAA concentrations. In the four obesity disease cats, their BW, BCS, plasma glucose and TP concentrations were not changed after anti-inflammatory compound supplementation for 4 weeks. Their TG, NEFA concentrations and LDH and ALT activities decreased. Plasma TC and SAA concentrations and AST activities decreased significantly in obesity disease cats after the compound supplementation. Their plasma adiponectin concentrations were maintained at low level, which were significantly lower than the control ranges $(4 \sim 6 \mu \mathrm{g} / \mathrm{mL})$. All four cats seemed to be alleviated from obesity disease as hyperlipidemia and high SAA concentrations were ameliorated.

\section{Discussion}

The main component of the anti-inflammatory compound, Rv-PEM01-99, is quercetin derivative. ${ }^{16,17}$ Quercetin is a flavonoid which has various functions such as antioxidant activity, anti-inflammatory action, amelioration of lipid metabolism and others. ${ }^{15,20,21}$ Quercetin supplementation is effective for improvement of lipid metabolism in obese animals. ${ }^{14,15,21}$ In this study, hyperlipidemia is ameliorated in the obesity disease cats after quercetin derivative supplementation for 4 weeks. Quercetin derivative supplementation does not reduce body weight and accumulated visceral fat in the obese cats, however, the supplementation possibly improves hepatic lipid metabolism and reduces plasma lipid (TG, T-Cho and NEFA) concentrations resulting in suppression of lipotoxicity caused by

Table I Comparison of Fasting Plasma Metabolites and Hormone Concentrations and Enzyme Activities in Healthy Cats Supplemented with and Without Rv-PEM0I-99 for 4 Weeks

\begin{tabular}{|l|l|l|l|l|}
\hline \multirow{2}{*}{} & \multicolumn{2}{|l|}{ Without Rv-PEM0I-99 $(\mathbf{n = 5})$} & \multicolumn{2}{l|}{ With Rv-PEM0I-99 (n=8) } \\
\cline { 2 - 5 } & $\mathbf{0}$ Week & $\mathbf{4}$ Week & 0 Week & $\mathbf{4}$ Week \\
\hline Body weight $(\mathrm{kg})$ & $3.3 \pm 0.2$ & $3.3 \pm 0.2$ & $3.4 \pm 0.2$ & $3.4 \pm 0.2$ \\
BCS & $5 / 9$ & $5 / 9$ & $5 / 9$ & $5 / 9$ \\
Glucose $(\mathrm{mg} / / 00 \mathrm{~mL})$ & $76.0 \pm 3.0$ & $78.4 \pm 4.0$ & $84.0 \pm 3.0$ & $78.3 \pm 1.3$ \\
TG $(\mathrm{mg} / / 00 \mathrm{~mL})$ & $38.5 \pm 2.0$ & $40.0 \pm 2.5$ & $39.0 \pm 2.5$ & $46.6 \pm 15.7$ \\
TC $(\mathrm{mg} / / 00 \mathrm{~mL})$ & $75.0 \pm 6.0$ & $88.0 \pm 7.0$ & $77.8 \pm 6.2$ & $95.9 \pm 6.6$ \\
NEFA $(\mathrm{mEq} / \mathrm{L}) 0.44 \pm 0.02$ & $0.44 \pm 0.02$ & $0.69 \pm 0.06$ & $0.73 \pm 0.09$ & $0.39 \pm 0.06^{*}$ \\
TP $(g / / 00 \mathrm{~mL})$ & $5.9 \pm 0.1$ & $6.1 \pm 0.2$ & $6.4 \pm 0.1$ & $6.3 \pm 0.1$ \\
MDA $(\mu \mathrm{mol} / \mathrm{L})$ & $1.49 \pm 0.15$ & $1.55 \pm 0.16$ & $1.89 \pm 0.15$ & $1.40 \pm 0.16^{*}$ \\
Adiponectin $(\mu \mathrm{gg} / \mathrm{mL})$ & $4.2 \pm 0.2$ & $4.6 \pm 0.4$ & $5.6 \pm 1.0$ & $5.8 \pm 0.6$ \\
LDH $(\mathrm{U} / \mathrm{L})$ & $182 \pm 20$ & $183 \pm 24$ & $218 \pm 9$ & $130 \pm 9^{*}$ \\
AST $(\mathrm{U} / \mathrm{L})$ & $26.5 \pm 1.3$ & $30.0 \pm 2.0$ & $27.5 \pm 1.3$ & $21.9 \pm 1.6$ \\
ALT $(\mathrm{U} / \mathrm{L})$ & $63.5 \pm 3.8$ & $57.0 \pm 3.2$ & $66.4 \pm 3.7$ & $56.5 \pm 4.8^{*}$ \\
SAA $(\mathrm{ng} / \mathrm{mL})$ & $15.9 \pm 5.2$ & $12.5 \pm 2.4$ & $19.2 \pm 4.2$ & $4.8 \pm 2.7^{*}$ \\
\hline
\end{tabular}

Notes: Values are presented as mean \pm SE. BCS is the average value of the group. *Significantly different $(p<0.05)$ from 0 week value in the same group.

Abbreviations: BCS, body condition score; TG, triglyceride; NEFA, non-esterified fatty acid; TP, total protein; MDA, malondialdehyde; LDH, lactate dehydrogenase; AST, aspartate aminotransferase; ALT, alanine aminotransferase; SAA, serum amyloid A. 
Table 2 Changes in Fasting Plasma Metabolites and Hormone Concentrations and Enzyme Activities in Four Obesity Disease Cats Supplemented with Rv-PEM0I-99 for 4 Weeks

\begin{tabular}{|l|l|l|l|}
\hline & 0 Week & 4 Week & P value \\
\hline Body weight $(\mathrm{kg})$ & $6.7 \pm 1.0$ & $6.6 \pm 1.0$ & 0.182 \\
BCS & $8.0 \pm 0.6 / 9$ & $8.0 \pm 0.6 / 9$ & - \\
Glucose $(\mathrm{mg} / 100 \mathrm{~mL})$ & $88.8 \pm 1.3$ & $91.3 \pm 11.3$ & 0.839 \\
TG $(\mathrm{mg} / 100 \mathrm{~mL})$ & $121.5 \pm 6.7$ & $85.0 \pm 10.9$ & 0.068 \\
TC $(\mathrm{mg} / 100 \mathrm{~mL})$ & $200.3 \pm 10.7$ & $167.3 \pm 18.4^{*}$ & 0.025 \\
NEFA $(\mathrm{mEq} / \mathrm{L})$ & $0.84 \pm 0.09$ & $0.66 \pm 0.02$ & 0.143 \\
TP $(g / 100 \mathrm{~mL})$ & $7.0 \pm 0.2$ & $6.9 \pm 0.2$ & 0.637 \\
MDA $(\mu \mathrm{mol} / \mathrm{L})$ & $2.3 \pm 0.0$ & $2.2 \pm 0.3$ & 0.628 \\
Adiponectin $(\mu g / \mathrm{mL})$ & $1.3 \pm 0.1$ & $1.2 \pm 0.1$ & 0.391 \\
LDH $(\mathrm{U} / \mathrm{L})$ & $204.0 \pm 53.0$ & $153.3 \pm 34.9$ & 0.118 \\
AST $(\mathrm{U} / \mathrm{L})$ & $47.0 \pm 4.7$ & $34.0 \pm 4.9^{*}$ & 0.006 \\
ALT $(\mathrm{U} / \mathrm{L})$ & $61.5 \pm 10.7$ & $49.0 \pm 11.3$ & 0.110 \\
SAA $(\mathrm{ng} / \mathrm{mL})$ & $194.3 \pm 76.0$ & $27.6 \pm 4.7^{*}$ & 0.004 \\
\hline
\end{tabular}

Notes: Data are expressed as mean \pm SE. $P$ value was calculated by paired $t$-test. *Significantly different $(p<0.05)$ from 0 week value.

Abbreviations: BCS, body condition score; TG, triglyceride; NEFA, non-esterified fatty acid; TP, total protein; MDA, malondialdehyde; $L D H$, lactate dehydrogenase; AST, aspartate aminotransferase; ALT, alanine aminotransferase; SAA, serum amyloid $\mathrm{A}$.

hyperlipidemia. $^{22}$ Previous reports found that the improved inflammatory microenvironment induced by quercetin may be involved in multiple signaling pathways, such as inhibiting the TLR/NF-kB signaling pathway and the PI3K/Akt/NF-kB/STAT3 pathway. ${ }^{13,23,24}$

In this study, reduction of plasma TG, TC and NEFA concentrations in the obesity disease cats was observed after quercetin derivative supplementation for 4 weeks. Longerterm supplementation with quercetin may be more effective on treatment for obesity disease. Obesity disease in cats is defined by overweight, low adiponectin, hyperlipidemia and high SAA concentrations. In this study, high plasma TC of $>180 \mathrm{mg} / 100 \mathrm{~mL}$ was considered as hyperlipidemia according to the criteria of metabolic syndrome in cats. ${ }^{8}$ Based on this amended criteria, all four cats with over $180 \mathrm{mg} /$ $100 \mathrm{~mL}$ of plasma TC are diagnosed as obesity disease, and they were alleviated from obesity disease after quercetin derivative supplementation.

Quercetin exerts an obvious anti-inflammatory activity in many animal species, ${ }^{25-27}$ and SAA as an inflammatory marker was reduced significantly in the obesity disease cats as well as in the healthy controls. MDA concentrations were not changed in the obesity disease cats, and this phenomenon may indicate insufficiency of anti-oxidant activity of quercetin derivative. Dose of quercetin derivative is adjusted to $2.53 .0 \mathrm{mg} / \mathrm{kg} /$ day, and at this dose, anti- inflammatory action was prominent in both healthy and obesity disease cats. This dose is low compared to the effective dose in dogs $(5.0 \mathrm{mg} / \mathrm{kg} /$ day $),{ }^{14}$ and this may indicate higher sensitivity of cats to quercetin compared to dogs. Effectivity against quercetin in cats is considered to be higher than in dogs. The supplementation period was only 4 weeks in this study, observing a short-term response. A long-term supplementation may be needed to show body weight reduction and antioxidant action as chronic effects as shown in mice. ${ }^{17}$ It is reported that high concentrations of quercetin show side effects to inhibit cytochrome $\mathrm{P} 450$ isoforms ${ }^{28}$ and metabolism of paclitaxel, an anticancer drug. ${ }^{29}$ Whereas, Rv-PEM01-99, quercetin derivative, does not show such side effects, ${ }^{16,17}$ therefore we can supply high concentrations of quercetin derivative to enhance its anti-oxidant and anti-inflammatory activities.

Quercetin supplementation does not reduce body weight and accumulated visceral fat in obesity disease cats, whereas the supplementation improves hepatic lipid metabolism resulting in decreased plasma lipid concentrations to alleviate obesity disease. An improvement of inflammatory condition is possibly caused by the antiinflammatory activity rather than the anti-oxidant activity of quercetin in the obesity disease cats.

This is a preliminary study to measure the effect of quercetin derivative in cats. Limitations of this study include small number of samples and biological and environmental variables (age, sex, diet), which is inevitable when client-owned animals from a private veterinary hospital are utilized. The obesity disease cats used in this study seemed to be in a mild stage of obesity disease without severe liver injury such as hepatic lipidosis as their plasma enzyme (LDH, AST and ALT) activities were not as high as those observed in metabolic syndrome. ${ }^{8}$ Supplementation of quercetin derivative at high dose (100 mg/kg/day) and for a longer period (8 weeks) causes a significant improvement of nonalcoholic fatty liver disease (NAFLD) by ameliorating inflammation, oxidative stress, and lipid metabolism in diabetic mice. ${ }^{13}$ Further studies are needed to clarify the effects of this anti-inflammatory compound supplementation in more cats with various severities of obesity disease and at different doses and durations of supplementation.

To alleviate obesity disease, a combination of the antiobesity food with low fat and high protein to reduce body weight with the anti-inflammatory compound like quercetin may be more effective in cats. 


\section{Conclusion}

Quercetin derivative seems to have strong anti-inflammatory activities. In the healthy cats, anti-inflammatory compound supplementation decreased plasma NEFA and SAA concentrations and LDH and ALT activities. In the obesity disease cats, the anti-inflammatory compound supplementation may alleviate obesity disease by relieving of inflammation and improvement of lipid metabolism in livers.

\section{Acknowledgments}

We would like to thank the staff of the Narita Animal Science Laboratory Co., Ltd., Chiba, for their reliable animal monitoring throughout the study period and taking blood samples from the animals, and Dr Laura Chadwick, Life Diagnostics, Inc., for kind provision of SAA measuring kits.

\section{Disclosure}

Kohei Suruga and Kazunari Kadokura are researchers working in Kibun Food Inc. The authors report no other conflicts of interest in this work.

\section{References}

1. World Health Organization. Obesity and overweight. 2019. Available from: http://www.who.int/mediacentre/factsheets/fs311/en/. Accessed January 30, 2020.

2. de Godoy MRC, Swanson KS. Companion animals symposium: nutrigenomics using gene expression and molecular biology data to understand pet obesity. J Anim Sci. 2013;91:2949-2964. doi:10.2527/jas.20 $12-5860$

3. Takahara M, Shimomura I. Metabolic syndrome and life style modification. Rev Endocr Metab Disord. 2014;15:317-327. doi:10. 1007/s11154-014-9294-8

4. Chandler M, Cunningham S, Lund EM, et al. Obesity and associated comorbidities in people and companion animals: a one health perspective. J Comp Pathol. 2017;156:296-309.

5. Tanaka A, Inoue A, Takeguchi A, Washizu T, Bonkobara M, Arai T. Comparison of expression of glucokinase gene and activities of enzymes related to glucose metabolism in livers between dogs and cats. Vet Res Commun. 2005;29:477-485. doi:10.1007/s11259-0051868-1

6. Mori A, Lee P, Takemitsu H, Sako T, Arai T. Comparison of insulin signaling gene expression in insulin sensitive tissues between cats and dogs. Vet Res Commun. 2009;33:211-226. doi:10.1007/s11259-0089168-1

7. Mori N, Okada Y, Tsuchida N, et al. Preliminary analysis of modified low-density lipoproteins in the serum of healthy and obese dogs and cats. Front Vet Sci. 2015;2:34. doi:10.3389/fvets.2015.00034

8. Okada Y, Kobayashi M, Sawamura M, Arai T. Comparison of visceral fat accumulation and metabolome markers among cats of varying BCS and novel classification of feline obesity and metabolic syndrome. Front Vet Sci. 2017;4:17. doi:10.3389/fvets.2017.00017

9. Kim S, Kim H. Inhibitory effect of astaxanthin o oxidative stress-induced mitochondrial dysfunction-a mini review. Nutrients. 2018;10:1137. doi:10.3390/nu10091137
10. Natoli R, Fernando N, Dahlenburg T, et al. Obesity-induced metabolic disturbance drives oxidative stress and complement activities in the retinal environment. Mol Vis. 2018;24:201-217.

11. Ribeiro MR, Lima RPA, Lisboa JVC. Influence of the C677T polymorphism of the MTHFR gene on oxidative stress in women with overweight or obesity: response to a dietary folate intervention. $J \mathrm{Am}$ Coll Nutr. 2018;27:1-8.

12. Otton R, Bolin AP, Ferreira LT, Marinovic MP, Rocha ALS, Mori MA. Polyphenol-rich green tea extract improves adipose tissue metabolism by down-regulating miR-335 expression and mitigating insulin resistance and inflammation. J Nutr Biochem. 2018;57:17 0-179. doi:10.1016/j.jnutbio.2018.03.024

13. Yang $H$, Yang $T$, Heng $C$, et al. Quercetin improves nonalcoholic fatty liver by ameliorating inflammation, oxidative stress, and lipid metabolism in $d b / d b$ mice. Phytotherapy Res. 2019;33:3140-3152. doi:10.1002/ptr.6486

14. Kawasumi K, Murai T, Mizorogi $\mathrm{T}$, et al. Changes in plasma metabolites concentrations in obese dogs supplemented with anti-oxidant compound. Front Nutr. 2018;5:74. doi:10.3389/fnut.20 18.00074

15. Murota K, Terao J. Antioxidative flavonoid quercetin: implication of its intestinal absorption and metabolism. Arch Biochem Biophys. 2003;417:12-17. doi:10.1016/S0003-9861(03)00284-4

16. Hiruma W, Suruga K, Kadokura K, et al. Antitumor effects and acute oral toxicity studies of a plant extract mixture containing Rhus verniciflua and some other herbs. Open J Immunol. 2015;5:39-49. doi:10.4236/oji.2015.51005

17. Suruga K, Tomita T, Kadokura K, Arai T. Rhus verniciflua leaf extract suppresses obesity in high-fat diet induced obese mice. Food Nutr Res. 2019;63:3601. doi:10.29219/fnr.v63.3601

18. Laflamme DP. Development and validation of a body condition score system for cats. A clinical tool. Feline Pract. 1997;25:13-18.

19. Okada Y, Ueno H, Mizorogi T, Ohara K, Kawasumi K, Arai T. Diagnostic criteria for obesity disease in cats. Front Vet Sci. 2019;6:284. doi:10.3389/fvets.2019.00284

20. Xu D, Hu M-J, Wang Y-Q, Cui Y-L. Antioxidant activities of quercetin and its complexes for medical application. Molecules. 2019;24 (6):1123. doi:10.3390/molecules 24061123

21. Guo W, Gong X, Li M. Quercetin actions on lipid profiles in overweight and obese individuals: a systematic review and meta-analysis. Curr Pharm Des. 2019;25:3087-3095. doi:10.2174/138161282566 6190829153552

22. DeFronzo RA. Insulin resistance, lipotoxicity, type 2 diabetes and atherosclerosis: the missing links. The Claude Bernard Lecture 2009. Diabetologia. 2010;53:1270-1287. doi:10.1007/s00125-0101684-1

23. Wang Z, Zhai D, Zhanf D, Bai L, Yao R, Yu C. Quercetin decreases insulin resistance in a polycystic ovary syndrome rat model by improving inflammatory microenvironment. Reprod Sci. 2017;24: 682-690. doi:10.1177/1933719116667218

24. Zhu M, Zhou X, Zhao J. Quercetin prevents alcohol-induced liver injury through targeting of PI3K/Akt/nuclear factor kappaB and STAT3 signaling pathway. Exp Ther Med. 2017;14:6169-6175.

25. Cui W, Hu G, Peng J, Mu L, Liu J, Qiao L. Quercetin exerted protective effects in a rat model of sepsis via inhibition of reactive oxygen species (ROS) and downregulation of high mobility group box 1 (HMGB1) protein expression. Mod Sci Monit. 2019;25: 5795-5800. doi:10.12659/MSM.916044

26. Johansson E, Lange S, Oshalim M, Lonnroth I. Anti-inflammatory substances in wheat malt antisecretory factor. Plant Foods Hum Nutr. 2019;74:489-494. doi:10.1007/s11130-019-00767-1

27. Hwang SH, Lorz LR, Yi DK, Noh JK, Yi YS, Cho JY. Viburnum pichinchense methanol extracts exerts anti-inflammatory effects via targeting the NF-kB and caspase-11 non-canonical inflammasome pathways in macrophages. J Endopharmacol. 2019;245:112161. doi:10.1016/j.jep.2019.112161 
28. Liu KH, Kim MJ, Jeon BH, et al. Inhibition of human cytochrome P450 isoforms and NADPH-CYP reductase in vitro by 15 herbal medicines, including Epimedii herba. J Clin Pharm Ther. 2006;31:83-91. doi:10.1111/j.1365-2710.2006.00706.x
29. Bun SS, Ciccolini J, Bun H, Aubert C, Catalin J. Drug interactions of paclitaxel metabolism in human liver microsomes. J Chemother. 2003;15:266-274. doi:10.1179/joc.2003.15.3.266

\section{Publish your work in this journal}

Veterinary Medicine: Research and Reports is an international, peerreviewed, open access journal publishing original research, case reports, editorials, reviews and commentaries on all areas of veterinary medicine. The manuscript management system is completely online

Submit your manuscript here: http://www.dovepress.com/veterinary-medicine-research-and-reports-journal and includes a very quick and fair peer-review system. Visit http://www.dovepress.com/testimonials.php to read real quotes from published authors. 